\title{
La educación social y los servicios sociales en los procesos de desarrollo comunitario: revitalización del trabajo en red
}

\section{Social education and social services in community development processes: revitalizing networking}

\author{
Laura Varela Crespo \\ UNIVERSIDAD dE SANTIAGO DE COMPOSTELA
}

\section{Resumen}

La comunidad es un contexto idóneo para la acción educativo-social que desarrollan las educadoras y educadores sociales en los servicios sociales comunitarios. El binomio constituido por la educación y el desarrollo requiere de la existencia de una comunidad en donde el vínculo que se teje entre ambas pueda manifestarse y hacerse efectivo. En este marco, el trabajo de las educadoras y educadores sociales necesita de la colaboración de otros profesionales que asuman un compromiso compartido en la planificación, desarrollo y evaluación de procesos educativos encaminados a potenciar la vida comunitaria a través de nuevos marcos de relación, como el representado por el "trabajo en red".

Palabras CLAVE: educación social, comunidad, desarrollo comunitario, servicios sociales comunitarios, trabajo en red.

\section{Summary}

The community is a suitable context for the educational-social action carried out by social educators in the so-called community social services. The coupling of education and development requires the existence of a community in which the link created between the two notions can manifest itself and become effective. In this setting, the work of the social educators needs the collaboration of other professionals that can take on a shared commitment to the planning, development and evaluation of educational processes designed to boost community life through new relationship frameworks, such as that represented by "networking".

KEY WORD: social education, community, community development, community social services, networking.

Nadie es sujeto en la soledad y el aislamiento, sino que siempre se es sujeto entre sujetos: el sentido de la vida humana no es un monólogo sino que proviene del intercambio de sentidos, de la polifonía coral. Antes que nada, la educación es la revelación de los demás, de la condición humana como un concierto de complicidades irremediables. (Savater, 2001: 34-35). 


\section{Introducción}

La comunidad, referente fundamental en la acción educativa, que se ha ido configurando en torno a la proximidad, la cooperación, la comunicación, el intercambio, etc., ha de constituir un eje central e ineludible del ámbito político-institucional más cercano a la ciudadanía, es decir, de la Administración Local. Ésta ha de actuar como "aseguradora" de los derechos sociales de las personas y encontrar en los servicios sociales comunitarios, en sus profesionales en general y en las educadoras y educadores sociales en particular, elementos clave para que se inicien, desarrollen y consoliden procesos educativos orientados al "empoderamiento" de los individuos y colectivos; a fin de que puedan tomar las "riendas" de sus propias vidas, las cuales se van (des)dibujando alrededor y en simultáneo con los procesos dinámicos, cambiantes, multidimensionales y de gran complejidad característicos de nuestra sociedad actual.

En este marco, y no dejando de reconocer la presencia y las actuaciones que en el seno de las comunidades desarrollan las entidades pertenecientes al denominado tercer sector, extendidas a lo largo y ancho de la geografía española, centraremos nuestra mirada en los servicios sociales comunitarios del sistema público, entendidos como agentes educativos dinamizadores de los territorios y comunidades de los que forman parte.

Los profesionales de los servicios sociales (trabajadores sociales, psicólogos, pedagogos, educadores sociales, etc.) han de trazar estrategias de trabajo coordinado que impliquen a las acciones comunitarias desde sus inicios (planificación), en sus procesos (implementación) y en los momentos de reflexión y reelaboración de las mismas (evaluación). Sin embargo, no sólo concierne a los profesionales trabajar de manera coordinada y co-responsabilizada, sino que a nivel interadministrativo tanto en sentido ver- tical (Administración estatal, autonómica, municipal) como horizontal (consejerías, departamentos, etc.) se requiere una apuesta firme por unos servicios sociales próximos de calidad; lo cual evidencia la necesidad de la transversalidad en las actuaciones que se llevan a cabo desde los distintos ámbitos de la política social (educación, vivienda, empleo, etc.), con el propósito de hacer efectiva la concepción de la persona como ser integral, en su doble dimensión individual y social.

\section{Repensar la comunidad desde la perspectiva de la educación social}

Han sido diversas las disciplinas que desde diferentes miradas, entre ellas la Psicología, la Sociología, la Pedagogía Social, la Antropología, han tomado a la "comunidad" como objeto de estudio. Se trata de un término complejo, polivalente, al que se le han otorgado numerosos significados. Entre otras, y revisando la literatura al uso, se identifican desde visiones utópicas e idílicas de la comunidad hasta alusiones a la comunidad como sinónimo de sociedad, pasando por referencias a la comunidad desligada de un ámbito territorial que la delimite (como es el caso de las comunidades virtuales) o con una delimitación muy precisa (comunidad de vecinos, comunidad escolar, etc.). Quizás, el significado más destacado sea el que evoca ese ideal de vida al que las personas aspiramos, sin saber con certeza cómo y cuándo podrá alcanzarse; ese paraíso perdido al que alude Bauman (2003), al que todos deseamos regresar.

Sin descuidar esta perspectiva de anhelo y utopía, en este trabajo nos referimos a la comunidad entendida, siguiendo a Caride, Pereira y Vargas (2007: 135), como "un espacio de vida social donde se configuran de forma constante múltiples y complejas relaciones e interacciones sociales entre individuos y colectivos que viven y conviven con 
lazos de solidaridad e intercambio de significados de su territorio, de su lengua y cultura y de sus vivencias individuales y comunes". En la comunidad así entendida, las relaciones e interacciones sociales entre cada individuo y los colectivos en los que se integra especialmente, se convierten en piezas fundamentales de los quehaceres cotidianos de la sociedad; en cuyo seno, la comunicación constituye un elemento clave, especialmente cuando la comunidad es observada e interpretada desde una mirada educativo-social.

Paulo Freire (1977) ya expresaba la relevancia de la interacción, indicando que es a través de las relaciones hombre-mundo como se construye el conocimiento, aludiendo también a la importancia del diálogo entendido como co-participación de los sujetos en el acto de pensar en búsqueda de la transformación social.

Las comunidades, en este marco, constituyen para las educadoras y educadores sociales contextos clave para la acción pedagógico-social, dado que si reconocemos que uno de los objetivos fundamentales de la Educación Social se refiere a generar procesos educativos que incidan en la mejora de la calidad de vida de la ciudadanía; la comunidad entendida como un contexto local, de proximidad y de interrelación entre diferentes individuos, instituciones, asociaciones, etc., es un escenario propicio y, en todo caso, ineludible para la acción comunitaria. Dicha acción es entendida, siguiendo a Úcar y Llena (2006: 38), como una forma de acción social, referida a las relaciones entre las personas, a todos aquellos mecanismos que, de una manera dinámica y compleja, entretejen, regulan y estructuran la vida en sociedad.

Se trata de una acción intencional, planificada, en la que participan diferentes agentes (ciudadanas y ciudadanos individualmente considerados, ciudadanía organizada -asociaciones de vecinos, clubes deportivos,
AMPA-, educadores sociales, trabajadores sociales, etc.) y cuyo referente clave es la emancipación y la transformación social. La participación, la concienciación, la educación, el empoderamiento (empowerment) y el desarrollo, son los cinco ejes alrededor de los cuales se estructuran y articulan en la actualidad las actividades, procesos y proyectos de la acción comunitaria.

La Administración Local, como el ámbito político-institucional de mayor proximidad a la ciudadanía, ha de asumir responsabilidades en la puesta en marcha de procesos educativos y participativos que contribuyan a que las personas se conviertan en protagonistas de sus propios procesos de desarrollo, tanto en la esfera individual como colectiva. De este modo, a pesar de las incesantes y vertiginosas transformaciones que han tenido lugar en las últimas décadas, como afirma Heras (2007), hay determinadas cuestiones en las que lo público sigue teniendo su obligación. Las administraciones públicas han de asegurar la garantía de los derechos de la ciudadanía, la protección de las personas que se encuentran en situación de vulnerabilidad, la cobertura a las necesidades básicas de los sujetos, etc.

Desde esta perspectiva, siguiendo a Subirats (2002), destacamos que el bienestar es cada vez menos una reivindicación global, para concretarse cada vez más en demandas personales y comunitarias, inscritas en la vida cotidiana. Los problemas y las expectativas generadas en y a través de las organizaciones primarias requieren soluciones específicas, basadas sobre todo en la proximidad.

En este marco, la educación debe ser entendida como una necesidad básica, esencial, además de un derecho de las ciudadanas y ciudadanos, lo que significa "abogar por satisfacer un derecho y cumplir una obligación o una responsabilidad antes de apelar a un recurso que actúa como "parcheo" para lavar la cara de un sistema socioeconómico que lleva inscritas sus propias lógicas excluyen- 
tes. Entender la educación como necesidad esencial, básica y común para todos, y asignarla al ámbito de los derechos y deberes de todos los miembros de la sociedad justa, tiene diversas implicaciones teóricas, prácticas y éticas" (Sáez, 2006: 209-210).

En definitiva, se trata de reconocer y alentar en las comunidades una mayor presencia de soportes políticos, estratégicos que favorezcan la implicación y la participación de las personas en la transformación de sus propias realidades. Implicación que permite pasar de la apatía a la movilización, pasar de la delegación y la dependencia a la actividad y la disponibilidad para asumir compromisos y riesgos. Participación que presupone la capacidad de utilizar los propios recursos, los propios poderes, ejerciendo la capacidad de decidir, de controlar. Pero, tanto la implicación como la participación necesitan de los elementos previos que la faciliten, como son la creación y existencia de conexiones entre personas, entre grupos presentes en la comunidad, y para ello es imprescindible el que se reconozcan intereses comunes y complementarios (Subirats, 2002: 36).

La educación en este proceso de cambio y transformación social, de "empoderamiento" de las personas está estrechamente ligada al desarrollo comunitario. En este sentido, la relación entre educación y desarrollo se caracteriza, según Caride y Vargas (2002), por tener lugar en las prácticas de la vida cotidiana, incluir a toda la población, abarcar todas las modalidades de educación recuperando la visión más "social" de la educación, poder realizarse con o sin mediación pedagógica especializada, presentar contenidos variados y diversos grados de intencionalidad, dar cabida a factores subjetivos y objetivos, no ser neutra, aplicar múltiples métodos pedagógicos, tener propósitos emancipatorios, transformar las realidades en la dirección de un desarrollo humano integral sustentable y equitativo, y promover la mejora de realidades concretas.
Al respecto, Longás y otros (2008) señalan que "si la educación no es compartida por los distintos agentes socioeducativos, no aborda y se nutre de la dimensión comunitaria y no genera desarrollo, cambio y transformación en las sociedades, no puede ser concebida como educación". En este sentido, la educación se convierte en un asunto de la comunidad al presentar como horizontes fundamentales el desarrollo humano y la transformación social. Es más, las características y el alcance de las relaciones que se establecen entre la educación y el desarrollo agrandan la concepción freiriana de la educación como una práctica transformadora entendida como "praxis, reflexión y acción del hombre sobre el mundo" (Freire, 1980: 7) para promover su emancipación y de las condiciones de existencia.

Esta relación entre educación y desarrollo como proceso de cambio y transformación social transcurre en torno a tres fases (Caride, Pereira y Vargas, 2007): la percepción e interpretación de los datos del mundo, la asimilación de los datos del mundo y la transformación del mundo. La primera fase, se refiere a la percepción sensorial de los fenómenos sociales que acontecen y la interpretación de los mismos para entender la realidad natural y social de forma comprensiva. La segunda, se centra en la lectura y asimilación por las personas de los mensajes captados, tras una reelaboración de los datos interpretados. Esta fase resulta fundamental en el desarrollo actitudinal de los individuos, de manera que en función de la interpretación que se elabore, las personas podrán adoptar actitudes conformistas o transformadoras. La tercera, se refiere a actuaciones que suponen cambios en el entorno, intencionales o no. Se trata de acciones que transforman el mundo, de muy diversos tipos, puesto que no hay un modo unívoco de producir cambios sociales.

En estas tres fases se manifiesta nítidamente la relación entre educación y de- 
sarrollo, y también la importancia de la existencia de una comunidad en donde este vínculo pueda concretarse y hacerse efectivo. La construcción del sentido de pertenencia, de unos intereses comunes, el establecimiento de interrelaciones, requieren necesariamente de procesos comunicativos y dialógicos que posibiliten compartir formas de pensar, de sentir y de actuar, a fin de trasladar el protagonismo de las instituciones a los verdaderos protagonistas; es decir, a las personas que configuran el complejo entramado de esa unidad que denominamos «comunidad».

Por todo ello, colegimos con Gomà y Brugué (2002: 79) al afirmar que la "educación debe satisfacer las necesidades educativas de la comunidad; no las de los decisores públicos. Y para hacerlo debe diseñarse desde abajo (...). En definitiva, es la comunidad la que puede definir los parámetros de la acción educativa o, incluso más, la que debe hacerlo si pretendemos que efectivamente educación y comunidad formen el binomio clave para el desarrollo personal y colectivo".

\section{Los servicios sociales comunitarios como agentes educativos de identidad local}

Los servicios sociales comunitarios, también denominados de atención primaria, básicos, generales, etc., constituyen el nivel más próximo a la ciudadanía en el sistema de servicios sociales. Desde nuestra perspectiva, entendemos los servicios sociales comunitarios como el conjunto coordinado de prestaciones, recursos, equipamientos, programas, etc., que posibilitan a todas las ciudadanas y ciudadanos ejercer su derecho a obtener una respuesta ante situaciones de necesidad, a recibir información y orientación frente a un problema o al desconocimiento de determinados recursos, a la promoción e integración social y a la participación en la vida cotidiana. A ellos se confía el logro de un mayor y mejor fortalecimiento de los lazos co- munitarios a través de equipos interdisciplinares que trabajan a favor de la calidad de vida, así como del bienestar individual y social de la ciudadanía.

En este sentido, la conceptualización y delimitación de los servicios sociales gira en torno a dos ejes fundamentales (March y Orte, 2004): por un lado, se basa en la consideración de los servicios sociales como un derecho de toda la ciudadanía, con actuaciones específicas dirigidas a los colectivos más desfavorecidos. Y por otro, en el diseño de estrategias orientadas al desarrollo de las personas, los grupos y las comunidades. Los servicios sociales aspiran a lograr la equidad social, valorando la diversidad y apostando porque cada persona pueda desarrollar su propio proyecto vital de manera autónoma. El elemento clave, según Subirats (2007), sigue siendo la lucha contra las desigualdades, pero reconociendo las diferencias, y con una fuerte defensa de la autonomía individual, entendida como una situación en la que no se produce la dominación de unas personas sobre otras.

Los servicios sociales caracterizados por su descentralización territorial y la tendencia a la universalización de las actuaciones para evitar la estigmatización, posibilitan la adopción y planificación estratégica de iniciativas orientadas a la potenciación de la vida comunitaria del territorio en el que se ubican. Como recurso de proximidad, participan en su vida cotidiana compartiendo los ritmos y tiempos diarios de gran parte de la ciudadanía; por ello, la acción educativa de las educadoras y educadores sociales, que trabajan tanto en el propio centro de servicios sociales como en el medio abierto, resulta imprescindible para que las personas que forman parte de la comunidad participen de manera activa en la resolución de los problemas, al tiempo que ha de contribuir al desarrollo de las habilidades y actitudes esenciales para adoptar, de manera autónoma, decisiones que redunden en su bienestar. 
Entendemos por medio abierto, siguiendo a Llena y Parcerisa (2008: 17), un "espacio de encuentro, que se ha construido y se va construyendo y reconstruyendo a partir de las interacciones entre los distintos componentes que lo forman: personas, instituciones, valores, objetivos y normas... En este contexto, la acción socioeducativa se caracteriza por su flexibilidad, por la posibilidad de autodeterminarse y de autoconstruirse colectivamente, por la voluntariedad en la participación y en la capacidad de decisión del conjunto de personas que conviven y se relacionan en este medio. Se trata de un medio educativo donde se parte de que la responsabilidad debe ser compartida por las personas que interaccionan".

Desde esta perspectiva, consideramos que el trabajo educativo en medio abierto de las educadoras y educadores sociales resulta primordial, dado que el desarrollo de una comunidad difícilmente puede realizarse desde el "espacio amurallado" en el que trabajan los profesionales. Es en la calle, en las plazas públicas, etc., donde la educadora o educador podrá iniciar una relación con las personas, especialmente con aquellos colectivos más vulnerables y desconocedores de los procedimientos burocráticos que se precisan para acceder a los servicios.

De ahí que, frente a las perspectivas asistencialistas que desde sus orígenes han caracterizado a los servicios sociales, frente a la actuación basada en síntomas y problemas, frente al predominio de la intervención individualizada, abogamos por unos servicios sociales entendidos como agentes educativos, como elementos de engarce de otros servicios existentes en la comunidad (centros educativos, sanitarios, culturales, etc.) que a través de diversos profesionales (psicólogos, educadores sociales, trabajadores sociales, etc.) lleven a cabo actuaciones conjuntas y coordinadas basándose en las necesidades y demandas de la población. Eso sí, sin obviar que estos servicios y sus pro- fesionales no son los únicos agentes de transformación, sino que los verdaderos agentes del cambio social son las personas con las que trabajan. Los servicios sociales comunitarios y las educadoras y educadores sociales han de dejar de ser quienes "dan" para que otros "reciban", facilitando que las ciudadanas y ciudadanos también puedan "dar" y "recibir" según sus propias circunstancias.

En este proceso de construcción de la dimensión educativa de los servicios sociales, podemos definir a la educadora o educador social de atención primaria, como "un profesional especializado en generar y acompañar procesos de cambio orientados a la autonomía y a la promoción de la persona (a fin de que ésta se convierte en protagonista de su propia vida), que genera y acompaña procesos educativos para promover la co- $^{-}$ nexión de la persona con la red social, que conoce y, en su caso, facilita el acceso a las ofertas formativas y recursos del territorio potencialmente educativos (escolares, culturales, de tiempo libre, etc.)" (Fité y Llena, 2000: 73). Se vuelve imprescindible, por tanto, que las educadoras y educadores sociales que trabajan en atención primaria conozcan todo aquello que tiene que ver con el medio en el que trabajan.

Sedó (1999), en este sentido, ensalza la importancia de que la educadora o educador esté ubicado en la comunidad, siendo necesario que complete su formación y experiencia como profesional, con un conocimiento profundo del territorio: equipamientos, tradiciones, dinámicas comunitarias, etc. La acción socioeducativa que desarrolla puede tener como destinatario, tanto al individuo, como a la familia o al grupo, partiendo bien de una demanda sentida y expresada, o bien de una demanda indirecta e institucional. En este último caso, es fundamental que la educadora o educador sea capaz de elaborar proyectos que respondan a las necesidades educativas y, de ser el caso, a las expectativas de las personas, para lo cual resultará clave 
que posea un conocimiento amplio de los sujetos y de la propia comunidad en la que lleva a cabo su actuación.

Asimismo, y vinculado al principio de universalidad que orienta a los servicios sociales comunitarios, abogamos porque las educadoras y educadores sociales trabajen con toda la población, especialmente con aquella más desfavorecida y vulnerable, exigiendo también la co-responsabilización de otras instituciones (familia, escuela, etc). Al tiempo, resulta fundamental la búsqueda de un equilibrio entre la prevención y la atención a las problemáticas que ya se han manifestado. Es aquí en donde se encuentra una de las principales tareas de la labor educativa orientada al desarrollo de la comunidad, pues la universalización de los servicios, la ruptura con la concepción todavía vigente de los servicios sociales entendidos única y exclusivamente como un instrumento de la política social al servicio de los colectivos más desfavorecidos, dificulta enormemente la puesta en marcha de procesos socioeducativos que cuenten con la implicación y la participación de todas las personas, y que contribuyan a la mejora de su calidad de vida.

La situación paradójica en la que se encuentran las educadoras y educadores sociales de atención primaria, actuando de "mediadoras" entre las demandas institucionales y las demandas de las personas y de la comunidad, requiere que la acción educativa se desarrolle en el marco de un equipo interprofesional de servicios sociales, siendo la educadora o educador quien asuma la coordinación de dicha actuación educativa. El profesional de la educación social no puede responsabilizarse individualmente del proceso de planificación de la acción socioeducativa, dado que las personas destinatarias de dicho proceso pueden presentar otras necesidades y demandas (de tipo psicológico, sanitario, laboral, etc.) que han de ser abordadas en colaboración, otorgándole un mayor protagonismo a otros profesionales.
En consecuencia, con el propósito de evitar la "fagocitación" de las actuaciones que se desarrollan desde los servicios sociales comunitarios, se hace imprescindible el establecimiento de un trabajo en red, coordinado por el equipo de servicios sociales de atención primaria de la zona. Es así que, siguiendo las interesantes propuestas de Silvia Navarro (2004: 121), nos orientemos a "pensar en red como alternativa a lo instituido, como posibilidad de generar un plano de consistencia donde la organización fija y estereotipada ceda su dominio a procesos de creatividad e invención que neutralicen otros de control y disciplinamiento. Pensar en red es una alternativa a la gestión de los riesgos, una estrategia que nos habilita a relacionar las producciones sociales y la subjetividad que en ellas se despliega".

\section{Nuevos marcos de interrelación en la comunidad: educación social, servicios sociales comunitarios y trabajo en red}

En el conjunto de documentos profesionalizadores del educador y educadora social, el Código Deontológico elaborado por ASEDES hace alusión al trabajo en red a través del principio de complementariedad de funciones y coordinación. Desde esta perspectiva, el educador o educadora ha de trabajar de forma coordinada, siendo consciente de su función dentro del equipo y de la posición que ocupa dentro de la red, y teniendo en cuenta que su actuación puede influir en el trabajo de los demás miembros que conforman el equipo. Dicha coordinación será fundamental a fin de que el resultado de las diferentes acciones socioeducativas que se llevan a cabo con la persona o el colectivo sea coherente y constructivo.

Pero si el educador o educadora social forma parte de un equipo, consecuentemente establecerá una serie de relaciones con quienes lo integran y con las dinámicas que emergen de su caracterización como 
un grupo de trabajo. Al respecto, la deontología profesional señala que: será consciente de su pertenencia al mismo y coherente con éste y con el proyecto socioeducativo de la institución en la que trabaje; será respetuoso con los miembros del equipo, antepondrá la profesionalidad a las relaciones afectivas con los compañeros del equipo; elaborará los aspectos educativos de los proyectos, que guíen las acciones socioeducativas de los profesionales que lo componen y contribuirá mediante su reflexión a que éstos se desarrollen; cooperará con el resto del equipo en la planificación, diseño, puesta en funcionamiento y evaluación de las acciones socioeducativas, así como en los mecanismos y criterios de transmisión de información; evitará interferir en las funciones, tareas o relaciones de los otros miembros del equipo con las personas sujetos de la acción socioeducativa; respetará y asumirá las decisiones del equipo tras ser contrastadas, argumentadas y acordadas, aún en el caso de que no las comparta, haciéndolas suyas a la hora de desarrollar la acción socioeducativa, siempre que la decisión del equipo no entre en contradicción con ninguno de los contenidos del código deontológico y se anteponga el interés de las personas, al de los profesionales; del mismo modo, informará al equipo o institución acerca de las irregularidades cometidas por algún miembro del equipo cuando perjudiquen la dignidad y el respeto de las personas en su ejercicio profesional.

Tomando como punto de partida estos referentes profesionalizadores, antes de profundizar en las características y potencialidades que se le atribuyen al trabajo en red, hemos de clarificar qué entendemos por red. Según el Diccionario de la Real Academia Española, en su vigésima segunda edición, la red es un conjunto de elementos organizados para un determinado fin. Pero ¿de qué elementos estamos hablando cuando nos referimos al trabajo en red en el seno de una comunidad?, ¿cuál es el fin pretendido?
Los integrantes de esa red son esencialmente los profesionales de los diferentes servicios (escuela, centro de salud, servicios sociales, equipamientos culturales, etc.), que hacen posible un trabajo coordinado y co-participado. Se trata de una actuación, que se inscribe dentro del trabajo comunitario, orientado "al desarrollo integral de un área territorial determinada y a la mejora de su nivel de vida, asistiendo y previendo las necesidades de la población, realizando actividades contra la marginación y la discriminación social, apoyando a sectores sociales desfavorecidos, y desarrollando tareas de dinamización social" (Caballo y otros, 1997: 87).

Por tanto, la red constituye un espacio de interrelaciones en donde los profesionales abordan las necesidades y demandas de las ciudadanas y ciudadanos desde una perspectiva transversal. En este sentido, siguiendo a Longás y otros (2008), pueden identificarse tres características fundamentales de las redes locales: la interprofesionalidad, la interdepartamentalidad y la interinstitucionalidad. La interprofesionalidad se refiere a la aportación del quehacer profesional de varios profesionales desde la complementariedad, lo que exige la realización conjunta del diagnóstico, de la definición de los objetivos y la metodología, de la evaluación, etc. La interdepartamentalidad alude al trabajo coordinado entre diferentes áreas o departamentos de una misma institución para asegurar una mayor eficiencia institucional. Y por último, la interinstitucionalización se define como la asunción de una responsabilidad compartida entre las administraciones y las instituciones, a fin de garantizar los derechos de la ciudadanía.

Estas tres características de la red son esenciales, dado que trabajar en convergencia con otros profesionales e instituciones posibilita caminar hacia el desarrollo integral de la comunidad, al facilitar la opti- 
mización de los recursos existentes en el territorio evitando solapamientos y duplicaciones en las actuaciones; al permitir la sincronización de los tiempos de los diversos servicios de la red, de manera que los horarios de los servicios puedan complementarse atendiendo a la realidad del territorio y no únicamente a las reivindicaciones laborales de los profesionales; al contribuir al aumento de la motivación de los trabajadores pudiendo compartir e intercambiar experiencias, problemas, sentimientos, dudas, etc., con otros profesionales que trabajan con las mismas personas o colectivos. En definitiva, posibilita llevar a cabo una actuación educativo-social entendida desde una perspectiva transversal.

En este sentido, y haciéndonos eco de las palabras expresadas por José Ramón Ubieto (2009) la pregunta que nos hemos de plantear no es si trabajamos en red, sino qué uso hacemos de la red. Las complejidades que caracterizan a la sociedad actual, planteando nuevos retos y desafíos, exigen inexorablemente el trabajo en red de los profesionales que participan en la acción comunitaria, de manera que se puedan proponer soluciones complejas y consensuadas a las múltiples caras que presentan los problemas sociales. Cada servicio constituye un nodo de la red general, partiendo de la corresponsabilidad de todos los profesionales que la conforman.

Uno de los retos del futuro, y también del presente, de los servicios sociales comunitarios entendidos como espacios de educación social estriba en otorgar a las personas y a la comunidad esa autonomía que les pertenece, pero que también han de construir, actuando la red como una "malla de seguridad" (Moreno, 2000) en la cual las personas encuentran el espacio que les es propio. Entretanto conseguiremos limitar la mal entendida "derivación" de los sujetos de un servicio a otro para no "situarlos a la deriva”, a merced de las circunstancias. Las respuestas impersonales de las instituciones sociales, que provocan la desaparición de la identidad de la persona, convirtiéndola en un "caso", en un expediente, deberán ser sustituidas por actuaciones educativas que contribuyan a la construcción y al fortalecimiento de las identidades de los sujetos y de la comunidad o comunidades en las que participan.

El educador o educadora social, por tanto, ha de actuar desde la mediación, entendida como el trabajo educativo que facilita un encuentro constructivo con otras personas, grupos, lugares, etc., siendo la finalidad última la emancipación de las personas en tanto que sujetos de la educación. De este modo, "los educadores-mediadores en los contextos institucionales han de poner énfasis en el ejercicio del diálogo, para dotar de fundamento pedagógico a aquellas acciones educativas destinadas al aprendizaje de habilidades comunicativas, como expresión de los valores cívicos de la educación social, entendida ésta como praxis de una democracia cultural y social que se sustenta en una pedagogía de lo cotidiano envolvente de toda la comunidad socioeducativa" (Caballo y Gradaílle, 2008: 48).

En este sentido, Silvia Navarro (2004: 66) recuerda que "sólo si cambiamos nosotros, si cambian las organizaciones desde las que intervenimos, conseguiremos también que cambie la comunidad para empezar a existir como tal, abandonando su rol de objeto por otro de sujeto activo. Sólo si dejamos espacio a la comunidad y la miramos, la reconocemos como tal, ésta empezará a encontrar espacio para, a partir de nuevas y apasionantes experiencias participativas, avanzar hacia aquel horizonte que en otros momentos las utopías hicieron ondear el viento".

\section{Reflexiones finales}

La palabra "comunidad", como modo de referirse a la totalidad de la población que 
habita en el territorio soberano del Estado, como ha indicado Bauman (2007), suena cada vez más vacía de contenido. Los vínculos humanos en la sociedad actual, caracterizada como sociedad red, modernidad líquida, sociedad del riesgo, etc., son cada vez más frágiles, inciertos y perecederos.

Los servicios sociales comunitarios que desempeñan funciones de información y orientación, de cooperación social, de ayuda a domicilio y apoyo a la unidad convivencial, de alojamiento alternativo, han de potenciar el desarrollo de la comunidad bajo el principio de universalización. La ciudadanía en general, y quienes se encuentran en situaciones de mayor incertidumbre e inestabilidad, los denominados "ciudadanos precarios" (Moreno, 2000), han de tener garantizado el derecho de poder disfrutar de estos servicios, a través de actuaciones educativas llevadas a cabo por equipos de profesionales de manera coordinada y co-responsabilizada. Se trata de una acción-intervención caracterizada por la complejidad, que siguiendo a Morin (2004), supone elementos aleatorios, azar, conciencia de las derivas y de las transformaciones.

En este ambiente inestable y de incertidumbre, el trabajo en red se presenta como una estrategia adecuada para caminar hacia metas comunes desde miradas interdisciplinares. Superar las diferencias y buscar elementos compartidos con otras instituciones y profesionales deviene en uno de los principales retos para las educadoras y educadores sociales que desde la atención primaria trabajan en procesos de desarrollo comunitario, o simplemente en la comunidad o con la comunidad.

La comunidad, tal y como la hemos definido, constituye un contexto idóneo para poner en marcha estos mecanismos y estrategias de coordinación e interrelación entre los profesionales de los servicios sociales de atención primaria y las demás instituciones y servicios que participan en la vida de la misma. Un contexto en el que será ineludible activar procesos de desarrollo comunitario que impliquen, siguiendo a Céspedes, Vegué y Blanco (2007), valores como la autonomía, la responsabilidad, el respeto y reconocimiento del otro. Se trata valores de naturaleza relacional que se construyen en la interacción "con el otro".

Se pretende, en definitiva, "el reconocimiento $y$, en su caso, transferencia de responsabilidades para las comunidades locales, para sus instituciones, para su capacidad organizativa, para las redes que articulan la vida cotidiana (movimientos de base, asociaciones, colectivos cívicos, etc.) y lo que ahora, enfáticamente se denomina sociedad civil. Entre otras medidas, creemos que se debe situar a las comunidades locales en el centro del desarrollo, partiendo de la realidad concreta de cada territorio y población, sin olvidar los "ruidos" (ventajas y desventajas) que acompañan a la globalización” (Caride, Pereira y Vargas, 2007: 126-127).

Desde esta perspectiva, las educadoras y educadores sociales han de constituir piezas clave en los procesos de planificación y actuación compartida y consensuada entre las personas que forman parte de la red, de manera que el individuo o el colectivo que participe en la acción socioeducativa que se lleva a cabo desde los servicios sociales sea considerado desde una óptica de desarrollo integral, teniendo la posibilidad de participar activamente en la comunidad a la que pertenece.

Frente a la visión de los servicios sociales vinculados al sistema de beneficencia, frente a los servicios sociales en los que el usuario es un mero receptor de servicios y prestaciones, consideramos de gran importancia la profundización en la dimensión educativa de los servicios sociales con el propósito de que los usuarios se conviertan en participantes, que el contexto de intervención se transforme en una comunidad concreta y en donde a través de las educadoras y educadores sociales como miem- 
bros de una red de profesionales e instituciones más amplia, se consigan poner en marcha procesos educativos que redunden en el desarrollo personal y social de la ciudadanía en general, y de quienes se encuentran en una situación de marginación y vulnerabilidad, en particular.

Los servicios sociales considerados como agentes educativos en el territorio en el que se ubican necesitan incluir en su conceptualización el binomio educación-desarrollo tratando de establecer relaciones equilibradas y complementarias entre las Administraciones Públicas, los equipos de profesionales que trabajan en el territorio y las ciudadanas y ciudadanos que componen las comunidades locales, a fin de que les sean reconocidas sus necesidades y demandas, sus potencialidades y dificultades, mediante el establecimiento y articulación de un trabajo en red consensuado y transversal. En el devenir de este proceso la educación social tiene mucho que ver, que decir y que hacer. Y debe hacerlo.

\section{Referencias bibliográficas}

ASEDES (2007): Documentos profesionalizadores. Barcelona: ASEDES. Disponible en: http://www.eduso.net/archivo/docdow.php?id=143 (fecha de acceso: noviembre 2009).

Bauman, Z. (2003): Comunidad. En busca de la seguridad en un mundo hostil. Madrid, Siglo XXI.

Bauman, Z. (2007): Tiempos líquidos. Vivir en una época de incertidumbre. Barcelona, Tusquets.

Caballo, M. B. y otros (1997): 131 conceptos clave da educación social. Santiago de Compostela: Universidade de Santiago de Compostela.

Caballo, M. B. y Gradaílle, R. (2008): "La Educación Social como práctica mediadora en las relaciones comunidad-escuela". Pedagogía Social. Revista Interuniversitaria, 15, pp. 45-55.

Caride, J. A. y Vargas, G. (2002): "La educación como desarrollo: una visión estratégica y humanista". Diálogos, 31-32, pp. 17-27.
Caride, J. A.; Pereira, O. y Vargas, G. (2007): Educaçao e desenvolvemento comunitario local. Perspectivas pedagógicas e sociais da sustentabilidade. Oporto, Profedições.

Céspedes, A.; Vegué, E.; Blanco, I. (2007): "Políticas sociales, educación y ciudadanía". Revista de Educación Social, $\mathrm{n}^{-}$6. Disponible en: http://www.eduso.net/res/?b=9\&c=78\&n=217 (fecha de acceso: noviembre de 2009).

Fité, M. y Llena, A. (coords.) (2000): I Jornades de Treball i Debat. L'educadora i l'educador social em l'atenció primaria. Barcelona, CEESC, Universitat de Barcelona.

Freire, P. (1977): La educación como práctica de la libertad. Madrid, Siglo XXI.

Freire, P. (1980): Extensión o comunicación. La concientización en el campo. Bogotá, Guadalupe.

Gomà, R. (2006): "L’acció comunitària: transformació social i construcción de ciutadania”. Quaderns d'Educació Social, 8, pp. 29-39.

Gomà, R. y Brugué, Q. (2002). "La educación en el marco de los servicios personales", en Subirats, J. (coord.): Gobierno local y educación. La importancia del territorio y de la educación en el papel de la escuela. Barcelona, Ariel, pp. 51-82.

Heras, P. (2007): "A educación social nos servizos sociais", en Cruz, L. (coord.): Actas da $2^{\underline{a}}$ escola de primavera. Educación Social e Servizos Sociais. Santiago de Compostela, CEESG, pp. 13-26.

Longás, J. y otros (2008): "Escuela, educación y territorio. La organización en red local como estructura innovadora de atención a las necesidades socioeducativas de una comunidad". Pedagogía Social. Revista Interuniversitaria, 15, pp. 137-151.

Llena, A. y Parcerisa, A. (coords.) (2008): La acción socioeducativa en medio abierto. Fundamentos para la reflexión y elementos para la práctica. Barcelona, Graó.

March, M. y Orte, C. (2004): "Os servizos sociais como contexto para a institucionalización e profesionalización do/a educador/a social", en AA.VV: Políticas socioeducativas. "Retos e propostas no século XXI". Santiago de Compostela, Xunta de Galicia, pp. 873-883.

Moreno, L. (2000): Ciudadanos precarios. La última red de protección social. Barcelona, Ariel. 
Morin, E. (2004): Introducción al pensamiento complejo. México, Gedisa.

Navarro, S. (2004): Redes sociales y construcción comunitaria. Creando (con) textos para una acción ecológica. Madrid, CCS.

Rafí, J. (2008): "Reflexiones desde la práctica”. Revista de Educación Social, n ${ }^{0}$ 7. Disponible en: http://www.eduso.net/res/?b=10\&c=91\&n=249 (fecha de acceso: noviembre de 2009).

Sáez. J. (2006): Pedagogía Social. Pensar la educación social como profesión. Madrid, Alianza.

Savater, F. (2001): El valor de educar. Barcelona, Ariel.

Sedó, C. (1999): Treballant com a educadora o educador social. Barcelona, Plenilluni.

Subirats, J. (coord.) (2002): Gobierno Local y Educación. La importancia del territorio y la comunidad en el papel de la escuela. Barcelona, Ariel.

Subirats, J. (dir.) (2007): Los Servicios Sociales de Atención Primaria ante el cambio social. Madrid, Ministerio de Trabajo y Asuntos Sociales.

Ubieto, J. R. (2009): El trabajo en red. Usos posibles en Educación, Salud Mental y Servicios Sociales. Barcelona, Gedisa.
Úcar, X. y Llena, A. (coords.) (2006): Miradas y diálogos en torno a la acción comunitaria. Barcelona, Graó.

DIRECCIÓN DE LA AUTORA: Laura Varela Crespo. Universidad de Santiago de Compostela. Departamento de Teoría de la Educación, Historia de la Educación y Pedagogía Social. Facultad de Ciencias de la Educación. Rúa Xosé María Suárez Núñez, s/n. Campus universitario sur. 15782. Santiago de Compostela.

Correo electrónico: laura.varela@usc.es

Fecha de recepción del artículo: 13.X.2008

Fecha de aceptación definitiva: 04.XI.2009

COMO CITAR ESTE ARTÍCULO:

Varela Crespo, L. (2010): "La Educación Social y los Servicios Sociales en los procesos de desarrollo comunitario: revitalización del trabajo en red". Pedagogía Social. Revista Interuniversitaria, 17, pp. 137-148. 